\title{
Solution and Bulk Properties of Branched Polyvinyl Acetates Part III-Intrinsic Viscosity and Light Scattering Measurements
}

\author{
G. C. BERRY*, L. M. HoBBS $\dagger$ and V. C. LonG $\ddagger$
}

\begin{abstract}
Light scattering and intrinsic viscosity measurements have been made on three different series of vinyl acetate polymers, one linear and two branched. One of the branched series was obtained by fractionation of a high conversion polymer, the other by a graft polymerization under conditions that allowed structural characterization of the resultant comb-shaped polymers. These data show that the ratio of the mean-square radii of gyration of branched and linear polymers of the same molecular weight is greater in good solvents than the ratio calculated ti eoretically for a theta solvent. The ratio of the second virial coefficients of the comb-shaped branched and linear polymers of the same molecular weight is equal within experimental error to the ratio of the intrinsic viscosities. The ratio of the intrinsic viscosities of branched and linear polymers is greater in a good solvent than in a theta solvent, in support of inferences from the behavior of the radius of gyration. A theoretical expression for the ratio of the intrinsic viscosities in a poor solvent is obeyed more nearly in a good solvent than in a theta solvent.
\end{abstract}

THE preparation of a linear and two series of branched poly(vinyl acetate) polymers has been described elsewhere ${ }^{1,2}$. The linear polymer, series 5 , was prepared by the careful fractionation of polymer that had been polymerized to low conversion at a low temperature using a photosensitive initiator $^{1}$. One of the branched samples, series 4 , was prepared in like manner from a polymer which had been polymerized to high conversion to give a randomly branched material ${ }^{1}$. The other, series 6 , was prepared by a graft polymerization technique to place a specified number of branches of predetermined average chain length on fractions of series 5 which served as a linear backbone polymer with a narrow molecular weight distribution ${ }^{2}$. The purpose of this paper is to consider some of the dilute solution properties of these branched polymers. In particular, the second virial coefficients, the mean square radii of gyration, and the intrinsic viscosities of the branched polymers in thermodynamically good solvents will be compared to the corresponding parameters for linear polymers. The melt viscosities of these branched polymers will be considered elsewhere.

* Present address: Mellon Institute, Pittsburgh. Pennsylvania.

t Presen: address: Bennettsvi!le, South Carolina.

†Present address: E. I. du Pont de Nemours and Co., Wilmington, Delaware. 
Intrinsic viscosity

Intrinsic viscosities were determined with the viscometers and techniques described in Part $\mathbf{I}^{\mathbf{1}}$.

The majority of the viscosity measurements were made on benzene solutions. The intrinsic viscosities for the linear polymers in benzene were fitted by the expression $[\eta]=k_{\eta}\langle M\rangle^{a}{ }_{w}$ with $k_{\eta}=2 \cdot 16 \times 10^{-4}$ and $a=0.675$. Sufficient measurements were made in 1,2,4-trichlorobenzene to establish the values of the constants to be $k_{\eta}=3.30 \times 10^{-4}$ and $a=0.623$. Intrinsic viscosities $[\eta]_{1}$ measured in benzene can be related to those $[\eta]_{2}$ in trichlorobenzene by the expression

$$
[\eta]_{2}=0.788[\eta]_{1}^{0-924}
$$

Equation (1) is used further below. The intrinsic viscosity data for the comb-shaped polymers, series 6 , are given in Table 1 . The data for the linear fractions, series 5 , and the randomly branched polymer, series 4 , have been given previously ${ }^{1}$.

Table 1. Intrinsic viscosity data

\begin{tabular}{|c|c|c|c|c|}
\hline Fraction* & Solvent & $\begin{array}{c}{[\eta]} \\
(d l / g)\end{array}$ & $k^{\prime}$ & $k^{\prime}+\beta$ \\
\hline $\begin{array}{l}6-20-1 \\
6-20-M \\
6-30-1 \\
6-30-M \\
6-40-1 \\
6-41-1 \\
6-41-M \\
6-50-1 \\
6-51-1 \\
6-51-M \\
6-60-1 \\
6-70-1 \\
6-71-1 \\
6-80-1 \\
6-81-1 \\
6-90-1 \\
6-91-1 \\
5-2-2 \\
5-5-3 \\
6-60-1 \\
6-70-1 \\
6-71-1 \\
6-80-1\end{array}$ & 2-Octanone & $\begin{array}{l}5 \cdot 20 \\
4 \cdot 18 \\
2 \cdot 96 \\
2 \cdot 77 \\
3 \cdot 18 \\
3 \cdot 64 \\
3 \cdot 34 \\
4 \cdot 52 \\
4 \cdot 48 \\
4 \cdot 15 \\
5 \cdot 76 \\
4 \cdot 00 \\
4 \cdot 32 \\
4 \cdot 27 \\
4 \cdot 04 \\
5 \cdot 01 \\
4 \cdot 19 \\
1 \cdot 35 \\
0 \cdot 940 \\
0 \cdot 950 \\
0 \cdot 953 \\
0 \cdot 616 \\
0 \cdot 696\end{array}$ & $\begin{array}{l}0.401 \\
0 \cdot 347 \\
0.366 \\
0.367 \\
0 \cdot 368 \\
0 \cdot 344 \\
0 \cdot 347 \\
0 \cdot 338 \\
0 \cdot 338 \\
0 \cdot 366 \\
0 \cdot 356 \\
0 \cdot 366 \\
0.355 \\
0 \cdot 369 \\
0.359 \\
0 \cdot 383 \\
0.381 \\
- \\
- \\
- \\
- \\
-\end{array}$ & $\begin{array}{l}0.501 \\
0.498 \\
0.502 \\
0.502 \\
0 \cdot 498 \\
0 \cdot 498 \\
0 \cdot 498 \\
0 \cdot 502 \\
0 \cdot 500 \\
0 \cdot 501 \\
0 \cdot 500 \\
0 \cdot 500 \\
0 \cdot 500 \\
0 \cdot 502 \\
0 \cdot 501 \\
0 \cdot 502 \\
0 \cdot 502 \\
- \\
- \\
- \\
- \\
-\end{array}$ \\
\hline
\end{tabular}

* Series 5 contains linear polymers.

Series 6 contains comb-shaped branched polymers.

\section{Light scattering}

Light scattering measurements were made at the instrument temperature of $34^{\circ}$ to $38^{\circ} \mathrm{C}$ in a Brice-Phoenix light scattering photometer with unpolarized $4358 \AA$ light and a narrow slit system. The photometer was calibrated with solutions of colloidal silica ('Ludox') and a solution of the Cornell standard polymer in toluene (conc. $0.5 \mathrm{~g} / \mathrm{dl}$ toluene). The cell was checked for symmetry with dilute fluorescein and the deviation from the 
Figure 1-Mean square radius of gyration versus molecular weight in trichlorobenzene: $\square$ combshaped, branched polymer, series 6; O linear polymer, series 5

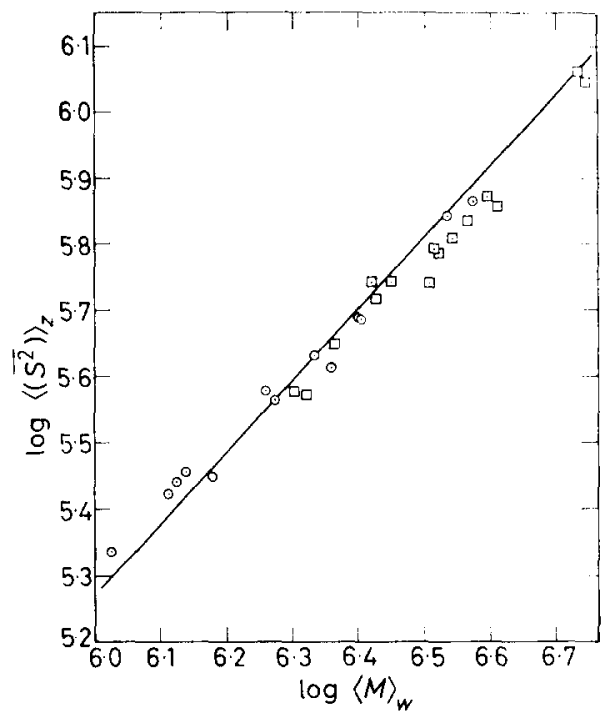

expected angular dependence was less than one per cent. Both solvent and solution were filtered into centrifuge tubes and centrifuged for about five hours to remove foreign matter. A dust free pipette fitted with a syringe needle and stopcock, and a device to draw the liquid slowly into the pipette by suction was used to remove the clarified liquid in some cases. A syringe with a manually operated plunger was used otherwise. Solvent clarity was judged by visual observation and by the ratio of the intensities of scattered light at $45^{\circ}$ and $135^{\circ}$ from the exit beam. In no case did this ratio exceed 1.05 . Solution clarity was judged visually and by the behaviour of the data. The same pipette was used to transfer the clarified solvent and the clarified solution. The relative intensity of the scattered light was measured at nine angles from $28^{\circ}$ to $135^{\circ}$. Butanone, 1,2,4-trichlorobenzene, and methanol were used as solvents. The latter two were distilled over calcium sulphate prior to use.

The data were treated in the usual manner to obtain a double extrapolation to zero angle and infinite dilution ${ }^{5}$. Thus, the data were plotted as $C / R_{\theta}$ versus $\sin ^{2}(\theta / 2)+$ (const.) $\times C$, where $R_{\theta}$ is the excess scattering ratio at $\theta^{\circ}$. The intercept gives $\langle M\rangle_{w}^{-1} \times K^{-1}$ where

$$
K=32 \pi^{3} n^{2}(\mathrm{~d} n / \mathrm{d} c)^{2} / 3 N \lambda_{0}^{4} \gamma_{n}
$$

Here $\gamma_{n}$ is a refraction correction for the solvent being used ${ }^{6}, n$ is the solvent refractive index, $\lambda_{i,}$ is the wavelength of the light used, $N$ is the Avagadro number and $\mathrm{d} n / \mathrm{d} c$ is the refractive index gradient. The $\mathrm{d} n / \mathrm{d} c$ values used for the three solvents were 0.080 for butanone ${ }^{7}, 0.1030$ for trichlorobenzene ${ }^{8}$, and 0.1319 for methanol ${ }^{9}$.

The reciprocal scattering curve, $P^{-1}(\theta)$ normalized to unity at $\theta=0$, was computed as $\lim _{c=0}\left(C / R_{\theta}\right)^{\prime}$ divided by the intercept. The initial slope of the curve multiplied by the factor $3\left(\lambda_{0} / 4 \pi n_{0}\right)^{2}$ gives the mean square radius of gyration averaged over chain lengths, this average being a $z$-average, $\left\langle\left(\overline{S^{2}}\right)\right\rangle_{z}$, in a theta solvent, and close to a $z$-average in a good solvent. The second 
virial coefficient, $A_{2}$, is obtained from the slope of the curve for $\lim _{\theta=0}\left(C / R_{\theta}\right)$. Since the initial slope of the reciprocal scattering curve is sometimes difficult to establish empirically, the method of $\mathrm{Zimm}^{5}$ was used to aid in its determination. The same procedure has arbitrarily been used to find the slopes of the $P^{-1}(\theta)$ curve for both the linear and branched polymer, although

Table 2. Light scattering data for the linear polymer in trichlorobenzene (TCB), butanone (MEK), and methanol (ME)

\begin{tabular}{|c|c|c|c|c|c|c|}
\hline Fraction & Solvent & $\langle M\rangle_{w} \times 10^{-6}$ & $\begin{array}{c}\left\langle\left(\overline{S^{2}}\right)\right\rangle_{z} \times \\
10^{4} A^{2}\end{array}$ & $\mid \begin{array}{c}A_{2} \times 10^{4} \\
\mathrm{~cm}^{3} \text { mole } / \mathrm{g}^{2}\end{array}$ & $\Phi_{l} \star \times 10^{-21}$ & $A_{2}\langle M\rangle_{w} /[\eta]$ \\
\hline $5-2-1$ & TCB & $3 \cdot 76$ & $73 \cdot 2$ & $1 \cdot 75$ & 1.86 & 155 \\
\hline $5-2-1$ & MEK & 3.58 & $93 \cdot 4$ & $2 \cdot 74$ & - & 175 \\
\hline $5-2-2$ & TCB & $3 \cdot 43$ & $69 \cdot 5$ & $1 \cdot 94$ & $1 \cdot 87$ & 176 \\
\hline $5-2-2$ & MEK & $3 \cdot 54$ & $66 \cdot 0$ & $1 \cdot 33$ & - & - \\
\hline $5-2-3$ & TCB & $2 \cdot 53$ & $49 \cdot 3$ & 1.90 & 1.78 & 157 \\
\hline $5-2-3$ & TCB & $2 \cdot 54$ & $48 \cdot 5$ & - & 1.84 & - \\
\hline $5-3-2$ & MEK & $2 \cdot 66$ & $68 \cdot 4$ & $2 \cdot 30$ & - & 123 \\
\hline $5-4-2$ & ТCB & $2 \cdot 27$ & $40 \cdot 8$ & 2.09 & $2 \cdot 20$ & 156 \\
\hline $5-4-2$ & MEK & $2 \cdot 28$ & $61 \cdot 5$ & $2 \cdot 64$ & - & 140 \\
\hline $5-4-1$ & TCB & $2 \cdot 16$ & $42 \cdot 5$ & 2.05 & 2.00 & 134 \\
\hline $5-5-2$ & TCB & 1.87 & $36 \cdot 4$ & 1.99 & 1.79 & 142 \\
\hline $5-5-2$ & MEK & 1.84 & $44 \cdot 0$ & $2 \cdot 62$ & - & 130 \\
\hline $5-5-2$ & ME & 1.90 & $30 \cdot 9$ & $1 \cdot 30$ & - & - \\
\hline $5-3-3$ & TCB & 1.81 & $37 \cdot 5$ & 1.98 & 1.79 & 126 \\
\hline $5-5-3$ & TCB & 1.50 & 28.0 & 1.90 & 1.82 & - \\
\hline $5-5-3$ & TCB & 1.33 & $27 \cdot 7$ & $2 \cdot 32$ & 1.71 & 132 \\
\hline $5-5-3$ & ME & 1.62 & $24 \cdot 4$ & $1 \cdot 14$ & - & - \\
\hline $5-6-1$ & TCB & $1 \cdot 29$ & $26 \cdot 3$ & $2 \cdot 39$ & 1.62 & 145 \\
\hline $5-6-1$ & TCB & $1 \cdot 38$ & $28 \cdot 5$ & $2 \cdot 29$ & 1.54 & 150 \\
\hline $5-6-2$ & MEK & $1 \cdot 14$ & $27 \cdot 2$ & $3 \cdot 42$ & - & 130 \\
\hline $5-6-3$ & MEK & 1.05 & $17 \cdot 2$ & $2 \cdot 35$ & $2 \cdot 16$ & 134 \\
\hline $5-6-3$ & MEK & 0.99 & 21.5 & $3 \cdot 38$ & - & 134 \\
\hline $5-7-2$ & MEK & 0.65 & $14 \cdot 6$ & $3 \cdot 26$ & - & 120 \\
\hline $5-7-4$ & MEK & 0.44 & 10.9 & 3.39 & - & 109 \\
\hline
\end{tabular}

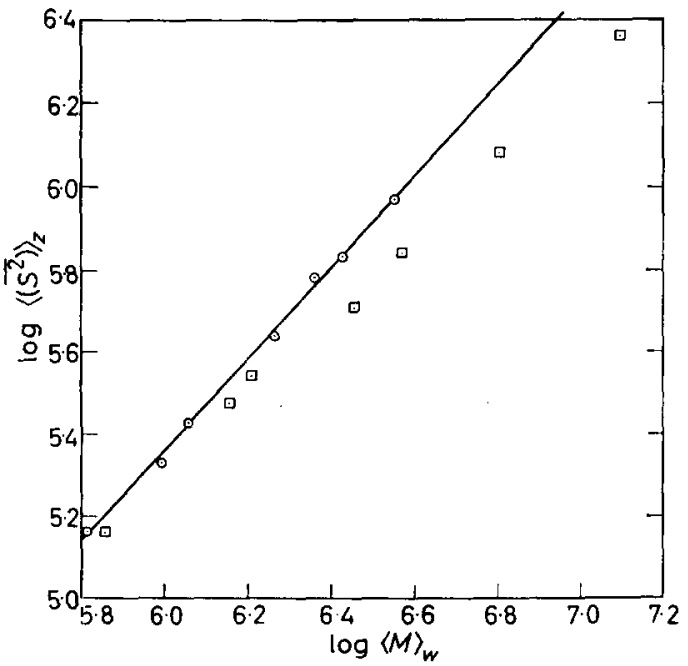

Figure 2-Mean square radius of gyration versus molecular weight in butanone: $\square$ randomly branched polymers, series 4; $O$ linear polymer, series 5 
Table 3. Light scattering data for the randomly branched polymer (series 4) and the comb-shaped polymer (series 6) in butanone (MEK), methanol (ME) and trichlorobenzene (TCB)

\begin{tabular}{|c|c|c|c|c|c|c|}
\hline Fraction & Solvent & $\langle M\rangle_{w} \times 10^{-6}$ & $\begin{array}{c}\left\langle\left(\overline{S^{2}}\right)\right\rangle_{z} \times \\
10^{4} A^{2}\end{array}$ & $\begin{array}{c}A_{2} \times 10^{4} \\
\mathrm{~cm}^{3} \text { mole } / \mathrm{g}^{2}\end{array}$ & $\Phi_{b} \star \times 10^{-21}$ & $S_{i} / S_{a}$ \\
\hline $4-1-1$ & MEK & $12 \cdot 6$ & 229 & 0.66 & - & - \\
\hline $4-2-3$ & MEK & $6 \cdot 43$ & 120 & $1 \cdot 37$ & - & - \\
\hline $4-3-2$ & MEK & $3 \cdot 76$ & $68 \cdot 8$ & $1 \cdot 13$ & - & - \\
\hline $4-4-1$ & MEK & $2 \cdot 86$ & $50 \cdot 8$ & $1 \cdot 33$ & - & - \\
\hline $4-4-2$ & MEK & 1.64 & $34 \cdot 5$ & $1 \cdot 78$ & - & - \\
\hline $4-5-1$ & MEK & $1 \cdot 43$ & $29 \cdot 9$ & 1.85 & - & - \\
\hline $4-4-3$ & MEK & 0.73 & $14 \cdot 5$ & $2 \cdot 39$ & 一 & 一 \\
\hline $4-5-2$ & MEK & 0.73 & $16 \cdot 6$ & $2 \cdot 99$ & - & - \\
\hline $4-5-3$ & MEK & $0 \cdot 43$ & $9 \cdot 1$ & $2 \cdot 72$ & - & - \\
\hline $6-20-1$ & TCB & $3 \cdot 81$ & - & - & - & - \\
\hline $6-20-M$ & TCB & $2 \cdot 80$ & $55 \cdot 4$ & 1.68 & 1.60 & 0.615 \\
\hline $6-30-1$ & TCB & $2 \cdot 33$ & - & - & - & - \\
\hline $6-30-M$ & TCB & 1.68 & - & - & - & - \\
\hline $6-30-M$ & TCB & 1.61 & - & 1.69 & - & - \\
\hline $6-40-1$ & TCB & $2 \cdot 00$ & $37 \cdot 5$ & $1 \cdot 78$ & $1 \cdot 60$ & 0.613 \\
\hline $6-41-1$ & TCB & $2 \cdot 24$ & $44 \cdot 2$ & 1.69 & 1.92 & 0.686 \\
\hline $6-41-M$ & TCB & 2.09 & $37 \cdot 2$ & 1.69 & 1.77 & 0.685 \\
\hline $6-50-1$ & TCB & $2 \cdot 71$ & $55 \cdot 1$ & $1 \cdot 78$ & 1.68 & 0.685 \\
\hline $6-51-1$ & TCB & $3 \cdot 67$ & $68 \cdot 7$ & 1.47 & 1.61 & 0.631 \\
\hline $6-51-M$ & TCB & $3 \cdot 21$ & $54 \cdot 3$ & 1.62 & 1.86 & 0.515 \\
\hline $6-60-1$ & TCB & $5 \cdot 60$ & $111 \cdot 5$ & 1.56 & 1.49 & - \\
\hline $6-60-1$ & TCB & $5 \cdot 43$ & $115 \cdot 8$ & $1 \cdot 18$ & $1 \cdot 38$ & 0.575 \\
\hline $6-60-1$ & ME & $6 \cdot 10$ & 99.6 & 0.99 & - & - \\
\hline $6-70-1$ & TCB & $2 \cdot 65$ & $52 \cdot 0$ & $1 \cdot 75$ & $1 \cdot 24$ & 0.623 \\
\hline $6-70-1$ & ME & 2.92 & $59 \cdot 8$ & 1.01 & - & - \\
\hline $6-71-1$ & TCB & $3 \cdot 43$ & $60 \cdot 1$ & $1 \cdot 29$ & $1 \cdot 77$ & 0.627 \\
\hline $6-80-1$ & TCB & $3 \cdot 29$ & $61 \cdot 3$ & $1 \cdot 52$ & 1.64 & 0.730 \\
\hline $6-80-1$ & ME & $3 \cdot 58$ & $59 \cdot 7$ & 1.06 & - & - \\
\hline $6-81-1$ & TCB & $4 \cdot 11$ & $71 \cdot 8$ & $1 \cdot 18$ & $1 \cdot 54$ & 0.615 \\
\hline $6-91-1$ & TCB & $3 \cdot 45$ & $64 \cdot 1$ & 1.48 & $1 \cdot 88$ & 0.715 \\
\hline $6-91-1$ & TCB & 3.96 & $74 \cdot 1$ & $1 \cdot 29$ & 1.46 & 0.624 \\
\hline
\end{tabular}

the assumptions in the derivation of the procedure are most appropriate to a linear polymer. The sharpness of the fractions and the insensitiveness of the slopes to the exact choice of the adjusting parameters probably make any errors introduced here of secondary importance. The light scattering data are given in Tables 2 and 3 . It is estimated that the experimental errors in $\langle M\rangle_{w}$, are approximately five per cent, and about ten per cent in both $\left\langle\left(\overline{S^{2}}\right)\right\rangle_{z}$ and $A_{2}$. Although these limits are estimates and not based on a statistical study, the several repeat runs shown give values which in general are within these limits.

\section{R E S U L T S}

Mean square radius of gyration

The mean square radius of gyration in trichlorobenzene, $\left\langle\left(\overline{S^{2}}\right)\right\rangle_{z}$, is shown as a function of $\langle M\rangle_{w}$ for the comb-shaped branched and linear polymers (Figure 1). Similar data for the randomly branched and linear polymers in butanone are shown in Figure 2. No attempt has been made to correct the radii to the weight average value, which would be more appropriate since 
a weight average molecular weight is used; the correction is neglected here since the shape of the curves and ratios to radii at constant $\langle M\rangle_{w}$ would not be altered by such a correction if all of the samples studied have about the same degree of polydispersity. The linear polymers are judged to have a low degree of polydispersity on the basis of the fractionation procedure ${ }^{1}$, and it was shown previously that the comb-shaped branched polymers should have about the same degree of polydispersity as the linear material used as a backbone polymer ${ }^{2}$. The randomly branched polymers are liable to be more polydisperse than the linear polymers, even though considerable. care was exercised in their fractionation. This is due to the dependence of the solubility on degree of branching as well as molecular weight. In the absence of a reliable estimate for the polydispersity of the randomly branched samples, we have assumed that they possess the same degree of polydispersity as the linear samples. The data for the linear polymers are fitted by the expression $\left(\overline{S^{2}}\right)=k_{s}\langle M\rangle_{w}^{b}$, with $b$ equal to 1.08 and $1 \cdot 12$ for trichlorobenzene and butanone, respectively, and $k_{s}$ equal to 0.062 for trichlorobenzene. It is evident that other values of the exponent may be used to fit the data with the same accuracy as those given here. The values given were chosen to be consistent with the Flory-Fox expression for the intrinsic viscosity. Even if this expression is not strictly valid in good solvents, the weak dependence of the expansion factor on molecular weight allows the method to be used here to good advantage since the intrinsic viscosity data are considerably more precise than those for the radius of gyration. Thus, the exponent $b$ is related to the exponent $a$ in the expression $[\eta]=k_{\eta}\langle M\rangle_{w}^{a}$ by $b=\frac{2}{3}(a+1)$, where $a$ is taken as 0.623 for polyvinylacetate in trichlorobenzene, and as 0.71 in butanone ${ }^{7}$.

The ratio of the radii for branched and linear polymers of the same molecular weight are given in Table 4. The ratio varies from about unity to values near 0.85 for the comb-shaped polymers, being generally somewhat lower for the randomly branched polymers. It is not easy to assess the errors in the ratio $\left\langle\left(\overline{S_{b}^{2}}\right)\right\rangle_{z} /\left\langle\left(\overline{S_{l}^{2}}\right)\right\rangle_{2}$. The ratios have been computed as. $\left\langle\left(\overline{S_{b}^{2}}\right)\right\rangle_{z} / k_{s}\langle M\rangle_{w}^{b}$ where $k_{s}$ and $b$ are the constants determined from the dependence of $\left\langle\left(\overline{S_{l}^{2}}\right)\right\rangle_{z}$ on $\langle M\rangle_{w}$, and $\left\langle\left(\overline{S_{b}^{2}}\right)\right\rangle_{z}$ and $\langle M\rangle_{w}$ are the measured parameters for the branched polymer. Thus errors in the ratio may be caused by errors in the determination of $\left\langle\left(\overline{S_{b}^{2}}\right)\right\rangle_{z}$ or $\langle M\rangle_{w}$, in the values. chosen for $k_{s}$ or $b$, or in the neglect of polydispersity corrections. The assumption that the branched and linear polymers have about the same degree of polydispersity is made with more confidence for the comb-shaped polymer than for the randomly branched polymer. We tentatively assign error limits of ten per cent to the ratio, recognizing that this may be a conservative estimate, and that this may influence conclusions to be drawn from the data.

\section{The intrinsic viscosity}

The effect of branching on the intrinsic viscosity of the polymers studied here may be seen in Figure 3. The intrinsic viscosity of the branched polymer is less than that of a linear polymer of the same molecular weight in all cases. The intrinsic viscosities have not been corrected for shear effects, since the data in Part $\mathrm{I}^{\mathrm{I}}$ indicated that comparisons of the intrinsic viscosities 
of branched and linear polymers at a given molecular weight are only negligibly dependent on shear effects. The ratios $\left[\eta_{b}\right] /\left[\eta_{l}\right]$ have been computed as $\left[\eta_{b}\right] / k_{\eta}\langle M\rangle_{w}^{a}$ where $k_{\eta}$ and $a$ are the constants determined from the dependenc of $\left[\eta_{l}\right]$ on $\langle M\rangle_{w}$, and $\left[\eta_{b}\right]$ and $\langle M\rangle_{w}$ are the parameters measured for the branched polymer. The primary source of error in this case is that in $\langle M\rangle_{w}$, so the ratio $\left[\eta_{b}\right] /\left[\eta_{l}\right]$ should be good to within five to seven per cent. The ratios $\left[\eta_{b}\right] /\left[\eta_{l}\right]$ are given in Table 4 .

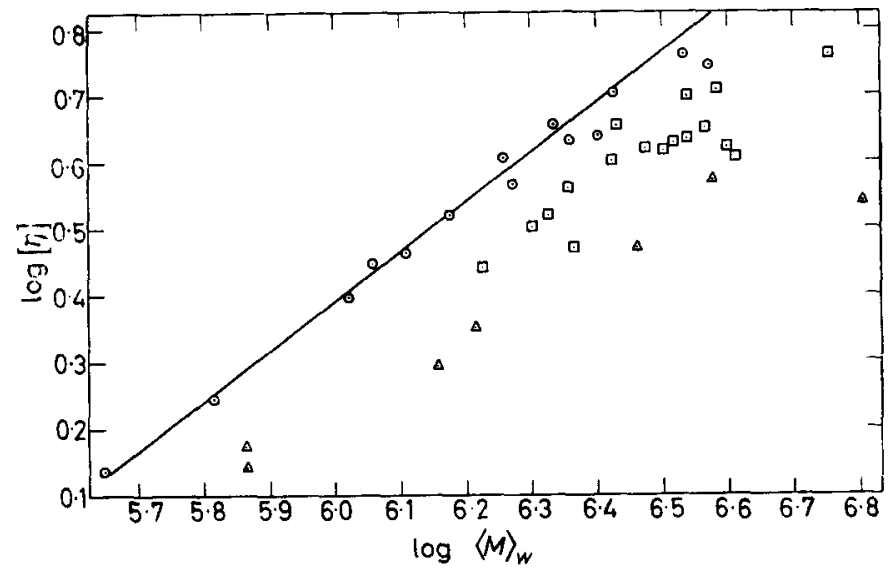

Figure 3-Intrinsic viscosity in benzene versus molecular weight:

$\square$ comb-shaped polymer, series 6 ; $\triangle$ randomly branched polymer, series 4; $O$ linear polymer, series 5

The second virial coefficient

Figure 4 gives $A_{2}$, the second virial coefficient, as a function of $\langle M\rangle_{w}$ for the linear and comb-shaped branched polymers. The data for the linear fractions are fitted by the expression $A_{2}=k_{A}\langle M\rangle_{x}^{-\gamma}$ where $\gamma$ and $k_{A}$ are

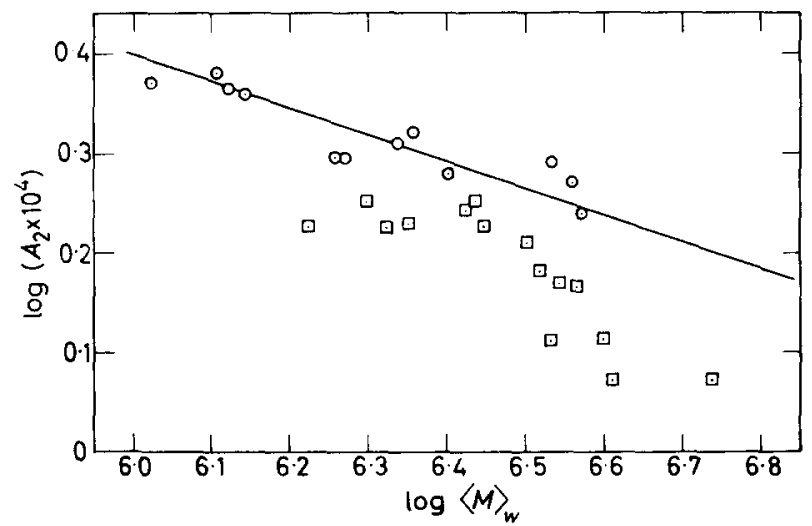

Figure 4-Second virial coefficient versus molecular weight in trichlorobenzene: $\square$ comb-shaped polymer, series 6 ; O linear polymer, series 5

0.230 and 0.00594 , respectively, for trichlorobenzene. The values of $\left(A_{2}\right)_{b}$ for the branched polymer are less than the corresponding values, $\left(A_{2}\right)_{1}$ for a 
linear polymer of the same molecular weight in every case. The ratio $\left(A_{2}\right)_{b} /\left(A_{2}\right)_{h}$ is given in Table 4 for the comb-shaped polymers. The $\left(A_{2}\right)_{b} /\left(A_{2}\right)_{l}$ have been computed as $\left(A_{2}\right)_{b} / k_{A}\langle M\rangle_{w}^{-\gamma}$, where $\left(A_{2}\right)_{b}$ and $\langle M\rangle_{w}$ are the parameters for the branched polymer and $k_{A}$ and $\gamma$ are the constants given above. The error in the ratio $\left(A_{2}\right)_{b} /\left(A_{2}\right)_{l}$ is due to experimental errors in both $\left(A_{2}\right)_{b}$ and $\langle M\rangle_{w}$, and is estimated to approximately ten to fifteen per cent.

Table 4. Parameters for the randomly branched polymer (series 4) and the combshaped polymer (series 6)

\begin{tabular}{|c|c|c|c|c|c|}
\hline Fraction & $\begin{array}{l}\text { Calc'd } \\
\quad g\end{array}$ & {$\left[\eta_{b}\right] /\left[\eta_{l}\right]$} & $\left\langle\left(\overline{S_{b}^{2}}\right)\right\rangle_{z} \mid\left\langle\left(S_{l}^{\overline{2}}\right)\right\rangle_{z}$ & $\left(A_{2}\right)_{b} /\left(A_{2}\right)_{l}$ & $\left(S_{i} / S_{\mathbf{a}}\right)_{b} /\left(S_{i} / S_{a}\right)_{l}$ \\
\hline $4-1-1$ & 0.32 & 0.345 & 0.580 & - & - \\
\hline $4-2-3$ & 0.36 & 0.395 & 0.648 & - & - \\
\hline $4-3-2$ & 0.54 & 0.615 & 0.678 & - & - \\
\hline $4-4-1$ & 0.50 & 0.590 & 0.681 & - & - \\
\hline $4-4-2$ & 0.58 & 0.658 & 0.858 & - & - \\
\hline $4-5-1$ & 0.55 & 0.625 & 0.870 & - & - \\
\hline $4-4-3$ & 0.62 & 0.705 & 0.891 & - & - \\
\hline $6-20-1$ & 0.616 & 0.842 & - & 0.836 & - \\
\hline $6-20-M$ & 0.821 & 0.821 & 0.966 & 0.858 & 0.880 \\
\hline $630-1$ & 0.556 & 0.659 & - & 0.614 & 一 \\
\hline $6-30-M$ & 0.766 & 0.773 & - & 0.769 & 0.734 \\
\hline $6-40-1$ & 0.750 & 0.786 & 0.949 & 0.844 & 0.876 \\
\hline $6-41-1$ & 0.670 & 0.936 & 0.986 & 0.804 & 0.686 \\
\hline $6-41-M$ & 0.718 & 0.804 & 0.890 & 0.807 & 0.978 \\
\hline $6-50-1$ & 0.744 & 0.897 & 1.01 & 0.908 & 0.980 \\
\hline $6-51-1$ & 0.524 & 0.738 & 0.896 & 0.799 & 0.902 \\
\hline $6-51-M$ & 0.641 & 0.738 & 0.823 & 0.860 & 0.734 \\
\hline $6-60-1$ & 0.671 & 0.703 & 0.924 & - & - \\
\hline $6-60-1$ & 0.690 & 0.703 & - & 0.709 & 0.794 \\
\hline $6-70-1$ & 0.777 & 0.804 & 0.969 & 0.884 & 0.886 \\
\hline $6-71-1$ & 0.630 & 0.737 & 0.853 & 0.694 & 0.883 \\
\hline $6-80-1$ & 0.693 & 0.748 & 0.904 & 0.809 & - \\
\hline $6-81-1$ & 0.553 & 0.666 & 0.834 & 0.701 & 0.878 \\
\hline $6-90-1$ & 0.736 & 0.848 & 0.898 & 0.797 & 1.02 \\
\hline $6-91-1$ & 0.641 & 0.671 & 0.902 & 0.714 & 0.896 \\
\hline
\end{tabular}

\section{I S C U S S I O N}

Mean square radius of gyration

It is of interest to compare the ratio of the radii of gyration of branched and linear polymers of a given molecular weight in a good solvent to the corresponding ratio calculated for theta-solvent conditions. It will be seen that the dimensions of the branched polymers increase at a faster rate than those of a linear polymer of corresponding molecular weight as the thermodynamic goodness of the solvent increases. First, however, we must determine the relative dimensions in a theta solvent for a basis of comparison. The latter may be computed if one assumes that random flight statistics are obeyed. This calculation has been given by Zimm and Stockmayer ${ }^{10}$ for a randomly branched polymer. The ratio may be obtained for the comb-shaped branched polymers, by use of the Kramers theorem ${ }^{11}$, which 
gives for a chain of $n$ equivalent segments

$$
g=\left(6 / n^{3}\right) \sum i(n-i)
$$

where $g$ is the ratio of the mean square radii for branched and linear polymers in a theta solvent. The sum is taken over all possible separations of the branched chain into two parts, containing $i$ and $(n-i)$ segments respectively. For purposes of this calculation, the branched polymer containing $n_{b}$ segments is assumed to have $k$ branches, each with $\left(n_{b}-n_{l}\right) / k$ segments, placed equidistantly on a backbone of $n_{l}$ segments. This idealization has negligible effect on the results for the kind of branched polymers of interest here. Thus, the sum of equation (3) for the comb-shaped polymer yields the result ${ }^{12}$

$g(k, x)=(1+k x)^{-3}\left\{(3 k-2) k x^{3}+(k+2) k x^{2}+\frac{2 k+1}{k+1} k x+1\right\}$

where $x$ is the ratio of the branch length to the backbone length, $\left(n_{b}-n_{l}\right) / k n_{l}$. For all of the comb-shaped polymers considered here $(x \leqslant 0.03)$, this expression is closely approximated by

$$
g \simeq 1 /(1+k x)=n_{l} / n_{b}
$$

Orofino ${ }^{13}$ has used a different technique to obtain an expression for $g$ for comb-shaped polymers with a random distribution of branch sites on the backbone, which reduces to equation (5) for the kind of branched polymers of interest here. It should be noted that the presence of a few branches attached to other branches rather than to the backbone, as discussed in Part II, will not affect the ratio $n_{l} / n_{b}$, and thus presumably have little effect on $g$, although it would require a separate calculation to prove this.

Values of $g$ can now be computed for each of the comb-shaped branched polymers of series 6 by use of equation (5) and the data reported in a previous paper ${ }^{2}$. These values are given in Table 4. The $g$ values are as reliable as the values of $\langle M\rangle_{w}$ for the comb-shaped polymer, and the backbone polymer. Thus, a primary source of uncertainty in $g$ is the molecular weight of the backbone polymer. The molecular weight of the polymer used as the backbone material is, of course, as well known as that of the final graft polymer. There remains, however, the possibility of a difference between the molecular weight of the initial and final backbone material due to selective loss of material in some of the necessary fractionations. We assume here in the calculation of $g$, that the average molecular weight of the backbone material is given by that of the initial polymer. (See ref. 2 for a further discussion of this point.) Any error in the backbone molecular weight will be carried over directly to the estimate of $g$. The effect of molecular weight polydispersity on $g$ is here neglected. The experimentally determined ratio of the radii is of course nearest to a $z$-average, while $g$ has been determined using weight average molecular weights. Orofino ${ }^{13}$ has given a form for $\langle g\rangle_{z}$ for a comb-shaped molecule which is useful in estimating the error made in computing $\langle g\rangle_{z}$ from equation (5) with weight average molecular weights, although not strictly applicable to these samples. The error will be negligible for all cases of interest here. 
It is evident from Table 4 that the values observed for $\left\langle\left(\overrightarrow{S_{b}^{2}}\right)\right\rangle_{z} /\left\langle\left(\overrightarrow{S_{l}^{2}}\right)\right\rangle_{z}$ for the comb-shaped polymers, series 6 , in the good solvent are greater than the calculated $g$, that is, the dimensions of the branched polymers have increased more than those of the linear molecules in passing from theta solvent to good solvent conditions. It is important to remember that the comb-shaped polymers should have about the same degree of polydispersity as the linear chains ${ }^{2}$, so that this difference should not be due to greater polydispersity in the branched polymer. A similar result is seen for the randomly branched polymers of series 4 , but here the values of $g$ must be estimated from viscosity data by a method discussed later, and the polydispersity of the branched chains might be greater than the linear polymers. An attempted empirical correlation of $\left.\left\langle\left(\overline{S_{0}^{2}}\right)\right\rangle_{z} /\left\langle\overline{S_{l}^{2}}\right)\right\rangle_{z}$ with $g$ is shown in Figure 5. The solid line drawn through the data is based on a theory to be described below. The lower dashed line shows where the data should lie if the ratio of the radii were equal in good and theta solvents.

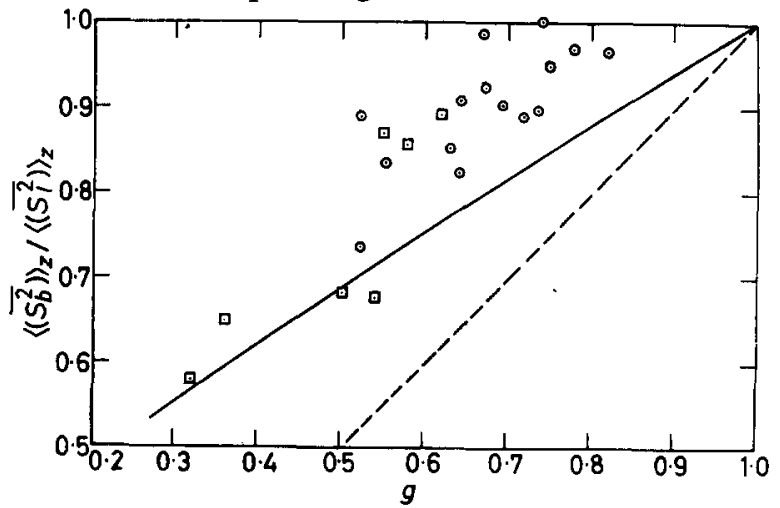

Figure 5-Ratio of the mean square radii of gyration for branched and linear polymers in a good solvent versus $g$, the same ratio calculated for a theta solvent: 0 combshaped polymers, series 6 , in trichlorobenzene; $\square$ randomly branched polymers, series 4 , in butanone. Broken curve is for the ratio in a good solvent equal to that in a theta solvent; the full curve is calculated from equation (11) with $C_{b}=C_{l}$

In order to facilitate discussion of the expansion effect observed here, it is convenient to introduce expansion factors, $\alpha$, defined as $\alpha^{2}=\left(\overline{S^{2}}\right) /\left(\overline{S^{2}}\right)_{0}$, where $\left(\overline{S^{2}}\right)$ and $\left(\overline{S^{2}}\right)_{0}$ are the mean square radii in thermodynamically nonideal and ideal (i.e. theta) solvents, respectively. The ratio $\left(\alpha, / \alpha_{l}\right)^{2}$ is then equal to $\left(\overline{S_{b}^{2}}\right) / g\left(\overline{S_{l}^{2}}\right)$, where the subscripts $b$ and $l$ refer to branched and linear polymers, respectively. Unfortunately, there are as yet no rigorous theories which allow prediction of $\alpha_{b} / \alpha_{l}$ for polymers in very good solvents. The first-order perturbation theory of Fixman ${ }^{14}$ may be used to obtain an expression for $\alpha_{b} / \alpha_{1}$ for both comb and star-shaped molecules, but the results are limited to solutions which are nearly ideal (to be published). The ratio $\alpha_{b} / \alpha_{l}$ is predicted to be greater than unity for molecules of these shapes in the limit as $\alpha$ approaches unity (in contradiction to Fixman's result for a four branched star, which is apparently in error). 
Ptitsyn ${ }^{15}$ has proposed an approximate derivation to account for the effect of branching on $\alpha$. The derivation, which closely follows Fixman's ${ }^{14}$ suggestion of a generalization of Flory's theory ${ }^{16}$ for linear chains, may be given as follows. It is assumed that the number of molecular configurations with radius of gyration $S$ in $\mathrm{d} S$ is given by

$$
F(S)=W(S) 4 \pi S^{2} \exp [-E(S) / k T]
$$

where $W(S)$ is the probability distribution of $S$ in the absence of volume effects, and $E(S)$ is the energy associated with $\rho(r)$, the segment density of the polymer chain at a distance $r$ from the centre of gravity. For a spherically symmetric $\rho(r), E(S)$ is given by

$$
E(S)=n^{2} \beta k T C / S^{3}
$$

where $\beta$ is the excluded volume integral for a pair of segments, and $C$ is a constant given by

$$
C=\frac{S^{3}}{2 n^{2}} \int 4 \pi r^{2} \rho^{2}(r) \mathrm{d} r
$$

The expansion coefficient may then be obtained ${ }^{17}$ by multiplying $F(S)$ by $S^{2}$ and integrating over all $S$. (The integration is not rigorous, but approximations are made according to techniques discussed in ref. 17). The result is

$$
\alpha^{5}-\alpha^{3}=n^{2} \beta C /\left(S^{2}\right)_{0}^{3 / 2}
$$

where $C$ is to be determined from equation (8). The familiar result of Flory ${ }^{16}$ obtains if a gaussian distribution is assumed for $\rho(r)$. If we assume that branched and linear chains are characterized by the same $W(S)$, but different $\rho(r)$, then

$$
\frac{\alpha_{b}^{5}-\alpha_{b}^{3}}{\alpha_{l}^{5}-\alpha_{l}^{3}}=\frac{C_{b}}{C_{l}}-\frac{1}{g^{3 / 2}}
$$

where $C_{b}$ and $C_{l}$ are the numerical constants computed from equation (8). Krigbaum and Trementozzi ${ }^{18}$ have pointed out that equation (10), with $C_{b}=C_{l}$, will be obtained directly from Flory's expression for $\alpha^{5}-\alpha^{3}$ for a linear polymer if this result is assumed to hold for branched chains with no change in certain parameters.

Unfortunately, the constants $C_{b}$ and $C_{l}$ are not readily obtained since the correct segment density distribution of the chain with excluded volume is not known. Ptitsyn assumes the same $\rho(r)$ for both types of chains, giving $C_{b}=C_{l}$. The ratio $C_{b} / C_{l}$ could be treated as a parameter to be determined by forcing agreement of equation (10) with the first-order perturbation theory of Fixman for a branched molecule. This procedure gives $C_{b} / C_{l}$ equal to 0.519 for a star molecule of four equal branches. Alternatively, the calculation can be altered by using more exact segment density distributions for the branched and linear chains. Thus, it is known that the sum of $n$ gaussian functions can correctly describe the segment density distribution of a random flight chain, though it is still in error for a chain with excluded volume $^{14,19}$, Casassa and Orofino ${ }^{20}$ have obtained $C_{l}$ using this approximation for $\rho(r)$, and it is possible to do the same calculation for a star- 
shaped polymer using the $\rho_{0}(r)$ given by Fixman and Stockmayer ${ }^{19}$. As for the linear chain, it is assumed that the $\rho(r)$ needed in equation (7) for the segment distribution in a good solvent is approximated by the $\rho_{0}(r)$ for a theta solvent with the modification that $\rho(r)$ is normalized to give the radius in a good solvent rather than in a theta solvent. We have done this calculation in the Appendix, and find the ratio $C_{b} / C_{l}$ is given by 1.09, 1.20 and 1.61 when the number of legs in the star-shaped molecule is equal to three, four and eight, respectively.

The reason for the difference between the ratio $C_{b} / C_{l}$ evaluated in these two ways is not clear. It may reflect inadequacies in the approximations made to obtain equation (10), or it may be that one simply cannot expect the constant obtained from the first order perturbation theory to be adequate for $a$ much larger than unity. It may be noted that values of $\alpha_{b} / \alpha_{l}$ determined from equation (10) differ only negligibly for low $\alpha$ for these two choices of $C_{b} / C_{l}$. We point out that equation (10) predicts that for a given $g$, the deviation of $\alpha_{b} / \alpha_{1}$ from unity will be strongly dependent on the deviation of $\alpha_{l}$ from unity. Thus a highly branched molecule in a solvent such that $\alpha_{l}$ is near unity should exhibit an $\alpha_{b} / \alpha_{l}$ ratio only slightly larger than unity.

In view of the discrepancies between these two treatments, and the lack of an estimate for the segment density distribution for comb-shaped or randomly branched polymers, we will tentatively compare equation (10) with $C_{b} / C_{l}$ equal to unity to the data of this study. Thus, the line drawn in Figure 5 has been obtained by setting $C_{b}=C_{l}$ in equation (10), and using the values of $x_{l}$ appropriate to the molecular weight of the polymers being studied. It appears that this function provides a reasonable fit to the data, although considerable scatter is evident and the data do not cover a sufficient range to adequately test the theory. Qualitatively, however, the data for the comb-shaped polymer suggest that $C_{b} / C_{l}$ might be somewhat greater than unity. The data can also be fitted within experimental error by the expression $\left(\overline{S_{b}^{2}}\right) /\left(\overline{S_{l}^{2}}\right) \simeq g^{0 \cdot 4}$, which is the limit of equation (10) with $C_{b}=C_{l}$ as $a$ becomes large. (Ratios of $\alpha_{b} / \alpha_{l}$ computed from equation (10) and from the limit of equation (10) for large $\alpha$ agree within about four per cent when $g$ and $\alpha$ are 0.6 and 1.9 respectively, so the limit is rapidly approached.) It should be noted, however, that in general one expects the deviation of $\left(\overline{S_{b}^{2}}\right) /\left(\overline{S_{l}^{2}}\right)$ from $g$ to depend on the solvent power, i.e. on some parameter such as $\alpha_{1}$. The observation that the branched polymers might be more extended in good solvents than linear polymers of the same molecular weight has been suggested by some previous studies ${ }^{21-24}$, but the uncertainties in polymer structure and the degree of polydispersity of the branched fractions complicated interpretation of the data. The data for the comb-shaped polymers provide evidence for the greater extension of the branched chains, at least for this type of structure. These data are supported by those on the randomly branched polymers, although here the specification of the structure and the degree of polydispersity is less certain. The values of $g$ (and hence of $\alpha_{b} / \alpha_{l}$ ) assigned to the branched polymers are obtained from a calculation based on their inferred structure rather han by direct measurements of the radii under theta solvent conditions. Thus, although the radii of the branched polymers are definitely 
larger than one would expect if the branched and linear molecules were equally expanded, the correct functional dependence of this effect is less certain. It should also be mentioned that these data could also be affected if some unknown impurity, such as hydroxyl groups, were present in the polymer although available evidence indicates the lack of such impurities ${ }^{2}$.

\section{The intrinsic viscosity}

It has been suggested that the ratio $\left[\eta_{b}\right]_{0} /\left[\eta_{l}\right]_{0}$, where $\left[\eta_{b}\right]_{0}$ and $\left[\eta_{l}\right]_{0}$ are the intrinsic viscosities of branched and linear polymers of the same molecular weight in a theta solvent, is a function only of the parameter $g$, written here as $f_{0}(g)^{19,24}$. One may reasonably expect this ratio in a good solvent to depend on $\alpha$ as well, that is $f(\alpha, g)$. It was originally suggested ${ }^{21}$ that $f_{0}(g)$ could be approximated by $g^{3 / 2}$ due to the dependence of the intrinsic viscosity on the volume of the polymer molecule. Fixman and Stockmayer ${ }^{19}$ and Zimm and Kilb ${ }^{25}$ have obtained theoretical estimates of $f_{0}(g)$, which attempt to account for hydrodynamic effects the above estimate ignores. Both of these derivations are based on star-shaped molecules. In particular, Fixman and Stockmayer have determined the effect of branching on the translational friction constant. It is then assumed that the ratio of the intrinsic viscosity of branched and linear polymers will be proportional to $h^{3}$, the corresponding ratio of the cube of the effective hydrodynamic radii, and that the form of the result will not be too dependent on the particular model chosen for the branched polymer. Thus, the result gives $f_{0}(g)=h^{3}$ where $h$ is a function of $g$ (see Figure 6). Zimm and Kilb have given a

Figure 6-Ratio of the intrinsic viscosities of combshaped branched and linear polymers versus $g$, the ratio of the radii of gyration calculated for a theta solvent. Curves $a, b$ and $c$ give $\left[\eta_{b}\right] /\left[\eta_{1}\right]$ equal to $g^{3 / 2}, h^{3}$ and $g^{1 / 2}$, respectively

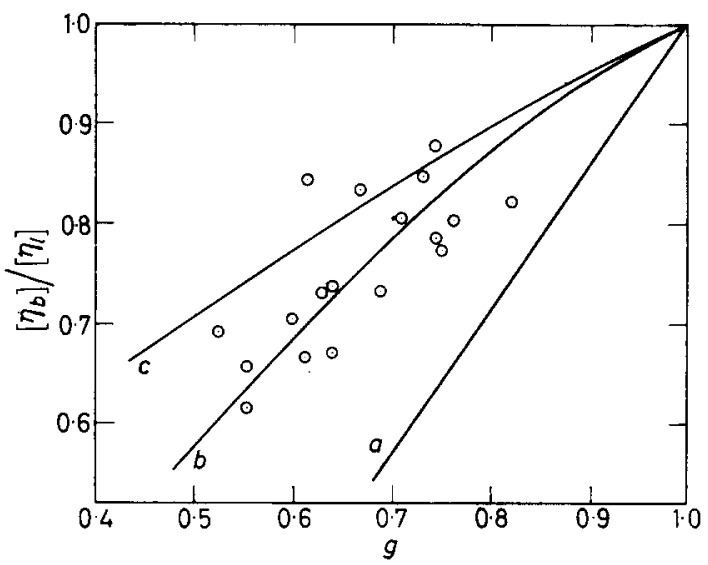

direct calculation of the intrinsic viscosity of a star-shaped molecule in a theta solvent taking approximate account of hydrodynamic interactions. Their results give $f_{0}(g) \simeq g^{1 / 2}$ if hydrodynamic interactions are included, and $f_{0}(g)=g$ if they are ignored. It is again assumed that the result is good for all types of branched polymers. It is to be emphasized that these results are limited to the behaviour of polymers in an ideal solvent. These functions are given in Figure 6 along with the experimental values of $\left[\eta_{b}\right] /\left[\eta_{l}\right]$ observed for the comb-shaped polymers in a good solvent as a function of $g$. The 
data are in general agreement with the theoretical functions $f_{0}(g)$ equal to $h^{3}$ or $g^{1 / 2}$. A similar result (not shown in Figure 6) is obtained with some data of Melville $e t$ al. ${ }^{26}$ on comb-shaped polymers, if equation (5) is used to compute $g$, and if the $[\eta] /\langle M\rangle_{w}$ relationship given above is used in place of that given by Melville. (The expression given by them leads to ratios of $\left[\eta_{b}\right] /\left[\eta_{l}\right]$ greater than unity for some of their data.) This correlation of the ratio $\left[\eta_{b}\right] /\left[\eta_{l}\right]$ determined in a good solvent with $f_{0}(g)$ is unexpected in view of the dependence of $[\eta]$ on the radius of gyration, and of the differences observed in the expansion factors for branched and linear polymers. Thus, the intrinsic viscosities of a linear polymer in a good solvent may be represented by the relationship

$$
\left[\eta_{l}\right]=6^{3 / 2} \Phi q_{i} \alpha_{l}^{m}{ }_{l}\left\langle\left(S_{l}^{2}\right)_{0}\right\rangle_{z}^{3 / 2} /\langle M\rangle_{w}
$$

where the subscript zero refers to parameters in an ideal solvent, $q$ is the polydispersity factor ${ }^{8}$ (taken to be identical for the linear and comb-shaped branched polymers), and $\Phi$ is a constant equal to $2.87 \times 10^{21}$

$$
\left[\eta_{b}\right]=6^{3 / 2} \Phi q_{b} \alpha_{b}^{m b}\left\langle\left(\overline{S_{b}^{2}}\right)_{0}\right\rangle_{z}^{3 / 2} f_{v}(g) / g^{3 / 2}\langle M\rangle_{w}
$$

( $[\eta]$ in units of deciliters per gramme, $\left(S^{2}\right)$ in $\left.\mathrm{cm}^{2}\right)$. We assume an analogous form for $\left[\eta_{b}\right]$, given by equation (12). Equation (11) has been derived on the basis of statistical calculations rigorously limited to polymer chains in very poor solvents ${ }^{27-29}$. The quantity $m_{b}$ in equation (12) is defined by the relationship $x_{b}^{m_{b}}=\left[\eta_{b}\right] /\left[\eta_{b}\right]_{0}$. Combination of equations (11) and (12) leads to the result (the branched and linear chains are assumed to have the same degree of polydispersity)

$$
\left(\left[\eta_{b}\right] /\left[\eta_{t}\right]\right) /\left(\left[\eta_{b}\right]_{0} /\left[\eta_{t}\right]_{0}\right)=\alpha_{b}^{m_{b}} / \alpha_{l}^{m_{t}}
$$

The value of $m_{l}$ is predicted to be about 2.5 for relatively good fractions of a linear polymer ${ }^{30}$. This predicted deviation of $m_{l}$ from the value of 3 given it in the Flory-Fox expression ${ }^{31}$ has been observed experimentally ${ }^{30,32-34}$. Since the statistical calculations leading to equation (11) are limited to poor solvents however, one can only treat $m_{l}$ as a parameter to be determined empirically in good solvents, its value probably being in the range 2.4 to 3 . If $m_{b}=m_{l}=m$, then equation (13) indicates that $\left[\eta_{b}\right] /\left[\eta_{l}\right]$ is greater than $\left[\eta_{b}\right]_{0} /\left[\eta_{1}\right]_{0}$ to the extent that $\left(\alpha_{b} / \alpha_{l}\right)^{m}$ exceeds unity. Some values of $\left[\eta_{b}\right]_{0}$ and $\left[\eta_{1}\right]_{0}$ were measured in 2-octanone at $29.8^{\circ} \mathrm{C}$, which is reported to be a theta solvent for linear polyvinyl acetates ${ }^{35}$, to allow this prediction to be checked. The data, which are given in Table 5, are seen

Table 5

\begin{tabular}{c|ccc}
\hline Fraction & $\begin{array}{c}{\left[\eta_{b}\right] /\left[\eta_{l}\right]} \\
\text { Observed } \\
\text { in benzene }\end{array}$ & $\begin{array}{c}{\left[\eta_{b}\right]_{0} /\left[\eta_{l}\right]_{0}} \\
\text { Observed } \\
\text { in 2-octanone } \\
\text { (theta solvent) }\end{array}$ & $\begin{array}{c}{\left[\eta_{b}\right] /\left[\eta_{l}\right]} \\
\text { Calculated } \\
\text { from eq. }(13) \text { with } \\
m_{b}=m_{l}=2.5\end{array}$ \\
\hline $6-60-1$ & 0.703 & 0.506 & 0.741 \\
$6-70-1$ & 0.804 & 0.745 & 0.983 \\
$6-71-1$ & 0.737 & 0.428 & 0.625 \\
$6-80-1$ & 0.748 & 0.498 & 0.699 \\
\hline
\end{tabular}


to show the trend expected from equation (13) with $m_{b}=m_{l}=2 \cdot 5$, although the agreement is not perfect. The discrepancy is probably caused by the error in determining the radii by light scattering, which would be sufficient to cause the scatter observed. Alternatively, it could be due to differences in the theta conditions for the branched and linear chains, to the assumption that $m_{b}=m_{b}$ or to errors in estimating $g$. The data of Thurmond and $\mathrm{Zimm}^{21}$ on the intrinsic viscosities of randomly branched polystyrene fractions in butanone and a theta solvent are of interest here since these data showed no appreciable deviation of $\left[\eta_{b}\right] /\left[\eta_{l}\right]$ from $\left[\eta_{b}\right]_{0} /\left[\eta_{l}\right]_{0}$. This is not unexpected, however, in view of the lower values of $\alpha_{l}$ for linear polymers of molecular weight corresponding to that of the branched polymer. Thus, if we use the estimated $\left(\alpha_{b} / \alpha_{l}\right)$ from equation (10) with $C_{b} / C_{l}$ equal to unity, then $\left[\eta_{b}\right] /\left[\eta_{l}\right]$ is found to exceed $\left[\eta_{b}\right]_{0} /\left[\eta_{l}\right]_{0}$ by only ten per cent for the branched polymer of highest molecular weight, becoming essentially unity for the lower fractions in their study.

It is also observed, however, as pointed out above, that the experimentally observed values of $\left[\eta_{b}\right] /\left[\eta_{l}\right]$ are in essential agreement with the theoretical function of $f_{0}(g)$ calculated from hydrodynamic theories, whereas the experimental values of $\left[\eta_{b}\right]_{0} /\left[\eta_{l_{0}}\right]_{0}$ are smaller than these theories would indicate. While it is true that the theoretical calculation of $f_{0}(g)$ is based on the assumption of a star-shaped molecule, this large $\alpha$ deviation of the experimental $f_{0}(g)$ from the value estimated from the theory is surprising. We do not know if the discrepancy indicates that results based on the starshaped model are inapplicable for the kinds of branched structure studied here, or if in fact there is a systematic error in the estimate of $g$ for the comb-shaped polymers.

In view of the agreement between the experimental data for $\left[\eta_{b}\right] /\left[\eta_{l}\right]$ and the theoretical function of Stockmayer and Fixman for $f_{0}(g)$ in those cases where $g$ could be determined from equation (5) and the known structural parameters, it was decided to use this function to obtain $g$ for the randomly branched polymers. The values of $g$ listed in Table 4 for these polymers were obtained in this manner.

\section{The second virial coefficient}

Rigorous expressions for $A_{2}$ in terms of molecular parameters have been developed for linear polymers ${ }^{36}$, but they are limited to solutions that are nearly ideal. The problem is, of course, less advanced for branched structures, but a rigorous calculation based on a cruciform molecule indicates that the ratio $\left(A_{2}\right)_{b} /\left(A_{2}\right)_{l}$ should be less than unity in very poor solvents ${ }^{19}$. Fixman and Stockmayer ${ }^{19}$ have suggested that the results of some approximate theories, which utilize an average potential obtained by an averaging over all configurations of two molecules separated by a given distance, may be useful in discussing the behaviour of branched polymers. The suggestion is based on their observation that the segment density distribution used in the averaging process is not too different for branched and linear polymers. The approximate theories give $A_{2}$ as a function of the parameter $\beta n^{2} /\left(\overline{S^{2}}\right)^{3 / 2}$, where the function decreases monotonically from unity as the argument increases from zero. Since the parameter $\beta$ is presumed to be independent of structural variations in a given polymer, 
differences in $A_{2}$ may be assigned to differences in the radius of gyration for branched and linear polymers. Thus, to the extent that $\left(\overline{S_{b}^{2}}\right)$ is less than $\left(\overline{S_{1}^{2}}\right)$ at a given molecular weight, $\left(A_{2}\right)_{b}$ will be less than $\left(A_{2}\right)_{l}$. In particular, Casassa and Markovit $z^{37}$ have given a function of this form which is useful here. It is of interest to note that values of $\left(A_{2}\right)_{l}$ calculated from this theory using our values for the radii in a good solvent and those of Schultz ${ }^{7}$ in a theta solvent are within twenty per cent of the observed values, but that the calculated values of $\left(A_{2}\right)_{l}$ are not quite as dependent on $\langle M\rangle_{w}$ as the observed values. This function approaches an asymptotic limit proportional to $\left(\overline{S^{2}}\right)^{3 / 2} / \beta n^{2}$ for values of the argument exceeding 3 or 4 . This expression may be incorrect even in the limit of very good solvents since this calculation, like all such treatments to date, does not treat terms due to multiple interaction contacts in a rigorous manner. It may be, however, that the ratio of the asymptotic expressions for $A_{2}$ for branched and linear polymers will not be too bad. To this approximation

$$
\left(A_{2}\right)_{b} /\left(A_{2}\right)_{l}=g^{3 / 2}\left(\alpha_{b} / \alpha_{l}\right)^{3}
$$

If the limit of equation (14) for large $\alpha$ is substituted into equation (15), then the ratio $\left(A_{2}\right)_{b} /\left(A_{2}\right)_{l}$ is approximated by $g^{1 / 2}$. A similar result has been noted by Krigbaum and Trementozzi ${ }^{18}$. Thus, the ratio $\left(A_{2}\right)_{b} /\left(A_{2}\right)_{l}$ should approximate the ratio $\left[\eta_{b}\right] /\left[\eta_{l}\right]$ since we find this to be near $g^{1 / 2}$. This is, in fact, close to the observed behaviour for the ratio, as seen in Table 4, indicating some degree of internal consistency to the data observed for the intrinsic viscosity, the virial coefficient and the radii of the branched polymer.

The values of $A_{2}$ for the linear polymers have been examined in terms of the dimensionless group $A_{2}\langle M\rangle_{w} /[\eta]$ which should increase with $M$ in the usual solvents and become constant for sufficiently good solvents. Table 2 gives this group as a function $\langle M\rangle_{w}$ for some linear fractions. The average value of $A_{2}\langle M\rangle_{w} /[\eta]$ is found to be 146 in trichlorobenzene and 134 in butanone, in good agreement to the values of 142 found by Chinai et al $^{38}$ in acetone, and 139 by Schultz ${ }^{7}$ in butanone. A slight trend with $\langle M\rangle_{w}$ is evident, however, indicating that the assumptions required for strict constancy of the group are not fulfilled.

\section{Reciprocal particle scattering curve}

In principle at least, the presence of branching can be detected from the shape of the reciprocal scattering curve if the size of the macromolecule is such that both the initial and asymptotic slopes of the curve are experimentally observable. It has been estimated that $\left(\overline{S^{2}}\right)$ should be in the range $10^{5}$ to $15 \times 10^{5} A^{2}$ for this condition to be fulfilled ${ }^{38}$. Calling $S_{i}$ and $S_{a}$ the initial and asymptotic slopes of the $P^{-1}(\theta)$ curve, respectively, then for a branched polymer ${ }^{\mathbf{1 2}, 40}$,

$$
\left(S_{t} / S_{a}\right)_{b}=(2 / 3)\left[\left\langle\left(\bar{S}_{b}^{2}\right)\right\rangle_{z} /\left\langle\left(\bar{S}_{l}^{2}\right)\right\rangle_{z}\right]\left[\langle M\rangle_{z} /\langle M\rangle_{w}\right] \mu \Gamma(\mu)
$$

where $\left(S_{l}^{2}\right)$ is the radius of a linear polymer of the same molecular weight as the branched polymer for which the $P^{-1}(\theta)$ curve is obtained, $\mu$ is a constant for a given polymer-solvent system, and $\Gamma$ is the gamma function. A similar expression written for the linear molecule has the term involving 
the ratio of the radii equal to unity. It was shown previously that the branched polymers prepared by the grafting procedure have about the same degree of polydispersity as the linear polymer used as the backbone molecule ${ }^{2}$. It is of interest, then, to compare the ratio of $\left(S_{i} / S_{a}\right)_{b}$ for these polymers to $\left(S_{i} / S_{a}\right)_{l}$ measured for the linear polymer used as the backbone. This ratio should be given by $\left\langle\left(S_{b}^{2}\right)\right\rangle_{2} /\left\langle\left(S_{l}^{2}\right)\right\rangle_{z}$. These data are given in Table 4. An appreciable scatter is evident, which is to be expected due to the uncertainty in determining the asymptotic slope of the $P^{-1}(\theta)$ curve, but a general agreement can be observed between the ratio $\left(S_{i} / S_{a}\right)_{b} /\left(S_{i} / S_{a}\right)_{i}$ and the ratio of the radii.

\section{The Flory constant}

The value of $\Phi \star$ in the expression $[\eta]=6^{3 / 2} \Phi \star S^{3} / M$ has frequently been used as an indication of the presence of branching. $\Phi \star$ is expected to be about $2.1 \times 10^{21}$ for linear polymers in the usual good solvents ${ }^{30,31}$. It has been pointed out by Kurata et al. ${ }^{28}$ that this is the value one expects from equation (11) if $\alpha_{l}$ and $m_{l}$ are about 1.4 and 2.5 respectively, and if one assumes the polymer is a reasonably good fraction. The best value of $\Phi_{1}^{\star}$ for the data in trichlorobenzene is about $2 \cdot 2 \times 10^{21}$, in good agreement with the expected value. Combination of equations (11) and (12) for polymers of the same molecular weight yields the result

$$
\frac{\Phi_{b}^{\star}}{\Phi_{l}^{\star}}=\frac{q_{b}}{q_{l}} \frac{f(\alpha, g)}{g^{3 / 2}}\left(\frac{x_{l}}{\alpha_{b}}\right)^{3}
$$

where $f(\alpha, g)$ is defined as $\left[\eta_{b}\right] /\left[\eta_{l}\right]$. If $\left(\alpha_{l} / \alpha_{b}\right)^{3}$ is approximated by the limit of equation (14) for large $\alpha$, and $f(\alpha, g)$ taken as $g^{1 / 2}$, then $\Phi_{b}^{\star} / \Phi_{t}^{\star}=q_{b} / q_{l}$. Thus, the ratio is dependent on the degrees of polydispersity of the branched and linear polymers if measurements are made in good solvents, rather than on the presence of branching. We note that the values of $\Phi^{\star}$ observed here were in general only slightly lower than the average for $\Phi \star$. In view of the deviation of $\alpha_{b} / \alpha_{l}$ from unity, it seems that the observation that $\Phi \star$ exceeds the normal value of $\Phi \star$ must in itself be regarded as an unreliable indication of the presence of branching.

\section{CONCLUSION}

We have presented data on two types of high molecular weight branched polymers, one of these polymers being synthesized in such a way that its characteristics are, in principle at least, well known. There are, of course, many factors which may cause difficulty in a study of this type, chemical impurities, slight gel content, polydispersity, to mention a few of the more serious complications. We have endeavoured to eliminate or account for these factors as effectively as possible. The data have been examined in terms of existing theories which are summarized in such detail as seemed necessary for the analysis. We may expect further studies on model branched polymers prepared by anionic methods, which offer some hope of eliminating or reducing the complications encountered here. It will be 
of interest, then, to examine data for polymers of different chemical constitution and of high enough molecular weight to give pronounced excluded volume effects to ascertain the universality of the effects found here.

We wish to thank the Goodyear Tire and Rubber Co. for their contribution to the Michigan Memorial Phoenix Project and the Allied Chemical and Dye Corporation for the financial assistance to one of us (G.C.B.) which made this work possible. We also wish to thank Professor $L$. $H$. Cragg of the University of Alberta, and Dr J. A. Manson for their helpful suggestion, and $\mathrm{Dr} C \mathrm{C} . \mathrm{H} . \mathrm{Lu}$ and $\mathrm{Mr} M . M$. Gurvitch for their aid with portions of the experimental work.

\section{Michigan Memorial Phoenix Project \\ and Chemical Engineering Department, \\ University of Michigan, Ann Arbor, Michigan}

(Received December 1962)

R E F E R E N C E S

${ }^{1}$ HoBss, L. M. and Long, V. C. Polymer, Lond. 1963, 4, 479

2 Berry, G. C. and Craig, R. A. Polymer, Lond. 1964, 5, 19

${ }^{3}$ Hobes, L. M., Kothari, S. C., Long, V. C. and Sutaria, G. C. J. Polym. Sci. 1956, 22, 123

4 Manson, J. A. and Cragg, L. H. J. Polym. Sci. 1958, 33, 193

${ }^{5}$ ZIMM, B. H. J. chem. Phys. 1958, 18, 1616

${ }^{6}$ CARR, C. L. and Zimm, B. H. J. chem. Phys. 1958, 18, 1616

${ }^{7}$ Shultz, A. R. J. Amer. chem. Soc. 1954, 76, 3422

${ }^{8}$ Graessley, W. W. Private communication

${ }^{9}$ Billmeyer, F. W. Private communication

10 Zimm, B. H. and StockmaYer, W. H. J. chem. Phys. 1949, 17, 1302

11 Kramers, H. A. J. chem. Phys. 1946, 14, 415

12 Berry, G. C. Ph.D. Thesis, University of Michigan, Ann Arbor, Michigan, 1960

13 Orofino, T. Polymer, Lond. 1961, 2, 295 and 305

${ }^{14}$ Fixman, M. J. chem. Phys. 1955, 23, 1656

15 PtrTsyn, O. B. and EIZnER, Y. E. Zh. fiz. Khim., Mosk. 1958, 32, 2464

16 Flory, P. J. J. chem. Phys. 1949, 17, 303

17 Grimley, T. B. Trans. Faraday Soc. 1954, 55, 681

18 Krigbaum. W. R. and Trementozzi, A. S. J. Polym. Sci. 1958, 28, 295

19 Fixman, M. and StockMAYER, W. H. Ann. N.Y. Acad. Sci. 1953, 57, 334

20 Casassa, E. F. and Orofino, T. A. J. Polym. Sci. 1959, 35, 553

21 Thurmond, C. D. and Zimm, B. H. J. Polym. Sci. 1952, 8, 477

22 Flory, P. J. and SchaefGen, J. R. J. Amer. chem. Soc. 1948, 70, 2709

23 Wales, M., Marshall, P. A. and Weissberg, S. G. J. Polym. Sci. 1953, 10, 229

24 Trementozzi, A. S. J. Polym. Sci. 1959, 36, 113

25 ZIMM, B. H. and KILB, R. W. J. Polym. Sci. 1959, 37, 19

26 Melville, H. W., Peaker, F. W. and Vale, R. L. J. Polym. Sci. 1958, 30, 29 and Makromol. Chem. 1958, 27, 140

27 Kurata, M. and Yamakawa, H. J. chem. Phys. 1958, 29, 311

28 Kurata, M., Yamakawa, H. and Teramoto, E. J. chem. Phys. 1958, 28, 785

29 Stockmayer, W. H. and Albrecht, A. C. J. Polym. Sci. 1958, 32, 215

30 Kurata, M., Yamakawa, H. and Utiyama, H. Makromol. Chem. 1959, 34, 139

${ }_{31}$ FLORY, P. J. and Fox, T. G. J. Amer. chem. Soc. 1951, 73, 1904 and J. Polym. Sci. $1950,5,745$

${ }^{32}$ SCHULZ, G. V. and KIRste, R. Symposium über Makromolekule (Wiesbaden 1959) 
${ }^{33}$ Zimm, B. H., OUter, C. I. and Carr, P. J. chem. Phys. 1950, 18, 830

${ }^{34}$ Krigbaum, W. R. and Carpenter, D. K. J. phys. Chem. 1955, 59, 1166

${ }^{35}$ Fox, T. G. Private communication

${ }^{36}$ ZIMM, B. H. J. chem. Phys. 1946, 14, 164

${ }^{37}$ Cassassa, E. F. and Markovitz, H. J. chem. Phys. 1958, 29, 493

38 Chinal, S. N., Scherer, P. C. and Leir, D. W. J. Polym. Sci. 1957, 17, 117

${ }^{39}$ Benoit, H., Holtzer, S. U. and Doty, P. M. J. phys. Chem. 1954, 58, 635

${ }^{40}$ Benort, H. J. Chim. phys. 1958, 55, 540

\section{APPENDIX}

We have to compute

where

$$
C=\frac{4 \pi}{2 N^{2}}\left[\frac{a^{2} N}{6}\left(\frac{3 \mathrm{f}-2}{\mathrm{f}^{2}}\right)\right]^{3 / 2} \int_{0}^{\infty} r^{2} \rho^{2}(r) \mathrm{d} r
$$

with

$$
\rho(r)=\left\{\pi^{-3 / 2} \sum_{n=0}^{N / 2} C_{n}^{3} \exp \left\{-C_{n}^{2} r^{2}\right\}\right.
$$

Thus

$$
C_{n}^{-2}=\frac{2 N a^{2}}{9 \mathrm{f}^{2}}\left[1+3(\mathrm{f}-2) \frac{\mathrm{f} n}{N}+3\left(\frac{\mathrm{f} n}{N}\right)^{2}\right]
$$

$$
\begin{gathered}
4 \pi \int_{0}^{\infty} r^{2} \rho^{2}(r) \mathrm{d} r=4 \pi \int_{0}^{\infty} r^{2} \sum_{j=0}^{N / t} \sum_{i=0}^{N / t} \mathrm{f}^{2} \pi^{-3} C_{j}^{3} C_{k}^{3} \exp \left\{-r^{2}\left(C_{j}^{2}+C_{k}^{2}\right)\right\} \mathrm{d} r \\
=\mathrm{f}^{2} \pi^{-3 / 2} \sum_{j=0}^{N / 1} \sum_{i=0}^{N / 1} \frac{C_{j}^{3} C_{k}^{3}}{\left(C_{j}^{2}+C_{k}^{2}\right)^{3 / 2}}
\end{gathered}
$$

Passing to the integral and transforming to reduced variables

$$
4 \pi \int_{0}^{\infty} r^{2} \rho^{2} r \mathrm{~d} r=\left(\frac{\pi 2 a^{2} N}{9 \mathrm{f}^{2}}\right)^{-3 / 2} N^{2} \int_{0}^{\mathrm{i}} \int_{0}^{1}\left[1+3(\mathrm{f}-2)(x+y)+3\left(x^{2}+y^{2}\right)\right] \mathrm{d} x \mathrm{~d} y
$$

The first integral is readily accomplished, and the second reduces to two standard integrals after the transformation

where

$$
y=\frac{K w}{\left(1-w^{2}\right)^{1 / 2}}-\frac{\mathbf{f}-2}{2}
$$

$$
K^{2}=(-1 / 12)\left(3 f^{2}-24 f+16\right)
$$

There results for $1 \leqslant \mathrm{f} \leqslant 3$

$$
\begin{aligned}
& \mathrm{f}=1,2, \quad C=\frac{1}{2} \cdot\left(\frac{3}{4 \pi}\right)^{3 / 2} \frac{4 \sqrt{ } 2}{3} \tan ^{-1}\left(\frac{3}{4}\right)=\frac{1}{2}\left(\frac{3}{4 \pi}\right)^{3 / 2} \times 1 \cdot 213 \\
& \mathrm{f}=3 \quad C=\frac{1}{2}\left(\frac{3}{4 \pi}\right)^{3 / 2} \times 1 \cdot 327
\end{aligned}
$$


and for $f \geqslant 4$

where

$$
\begin{gathered}
C=\frac{1}{2}\left[\frac{3(3 \mathrm{f}-2)}{4 \pi}\right]^{3 / 2} \frac{1}{3 \sqrt{ } 2 u} \ln \left\{\left(\frac{3 \mathrm{f}(\mathrm{f}-2)+2 \sqrt{ } 2 \sqrt{ }(3 \mathrm{f}-1) u}{3 \mathrm{f}(\mathrm{f}-2)-2 \sqrt{ } 2 \sqrt{ }(3 \mathrm{f}-1) u}\right)^{2}\right. \\
\left.\left(\begin{array}{l}
3 \mathrm{f}^{2}-4 \sqrt{ }(3 \mathrm{f}-2) u \\
3 \mathrm{f}^{2}+4 \sqrt{ }(3 \mathrm{f}-2) u
\end{array}\right) \times\left(\frac{3(\mathrm{f}-2)^{2}-4 u}{3(\mathrm{f}-2)^{2}+4 u}\right)\right\}
\end{gathered}
$$

Thus

$$
u=\sqrt{ }\left(3(f-2)^{2}-4\right)
$$

$$
\begin{aligned}
& \mathrm{f}=4 ; \quad C=\frac{1}{2}\left(\frac{3}{4 \pi}\right)^{3 / 2} \times 1.413 \\
& \mathrm{f}=8 ; \quad C=\frac{1}{2}\left(\frac{3}{4 \pi}\right)^{3 / 2} \times 1.594 \\
& \lim _{\mathrm{f} \rightarrow \infty} \quad C=\frac{1}{2}\left(\frac{3}{4 \pi}\right)^{3 / 2} 8\left(1-\frac{1}{\sqrt{ } 2}\right)
\end{aligned}
$$

